\title{
A tensorial toolkit for quantum computing in lattice gauge theory
}

\author{
Yannick Meurice ${ }^{* \dagger}$ \\ University of Iowa \\ E-mail: yannick-meurice@uiowa.edu
}

In most lattice simulations, the variables of integration are compact and character expansion (for instance Fourier analysis for U(1) models) can be used to rewrite the partition function and average observables as discrete sums of contracted tensors. These reformulations have been used for RG blocking but they also naturally fit the needs of quantum computing. We discuss FAQ about tensorial reformulations: effects of truncations on symmetries, boundary conditions, Grassmann variables, and other recent aspects of quantum computing in the same context.

The 36th Annual International Symposium on Lattice Field Theory - LATTICE2018

22-28 July, 2018

Michigan State University, East Lansing, Michigan, USA.

\footnotetext{
* Speaker.

${ }^{\dagger}$ This research was supported in part by the Dept. of Energy under Award Numbers DE-SC0010113 and DESC0019139. 


\section{Introduction}

Lattice QCD has been very successful at establishing that QCD is the theory of strong interactions, however some aspects remain inaccessible to classical computing: some finite density calculations have a sign problems and real time evolution requires detailed information not available from conventional MC simulations at Euclidean time. For these reasons, there has been a recent interest to consider the experimental use of cold atoms [1,2] or trapped ions [3] to perform quantum simulations relevant for lattice gauge theory and high energy physics. For reviews of various aspects of this approach, see Refs. [4, 5, 6]. A complementary approach is to use existing quantum computers $[7,8,9,10]$. A long term and ambitious goal that could have a significant impact on our understanding of collider physics would be to do jet physics calculations from first principles.

The tensor renormalization group (TRG) approach of lattice models provide tools that naturally fit the needs of quantum computations/simulations $(\mathrm{QC} / \mathrm{S})$. In most lattice models, the variables of integration are compact and character expansions (such as Fourier series) can be used to rewrite the partition function and average observables as discrete sums of contracted tensors. As an example for the $O(2)$ model, we have for each link:

$$
\mathrm{e}^{\beta \cos \left(\theta_{i}-\theta_{j}\right)}=\sum_{n_{i j}=-\infty}^{+\infty} \mathrm{e}^{i n_{i j}\left(\theta_{i}-\theta_{j}\right)} I_{n_{i j}}(\beta)
$$

This reformulations have been used for RG blocking but they are also suitable for QC/S when combined with appropriate truncations. In section 2, we review some aspects of the TRG approach in this context. In section 3, we discuss Frequently Asked Questions (FAQs) about tensorial formulations: 1) effects of truncation on global symmetries, 2) boundary conditions and 3) the use of Grassmann tensors for the Ising model. In section 4, we comment on various questions discussed during the conference and after: analog versus digital computations and the treatment of noncompact fields.

\section{The Tensor Renormalization Group method}

The Tensor Renormalization Group (TRG) $[11,12,13]$ is an implementation of Wilson program for lattice models with controllable approximations [14]. It leads to universal fixed point equations, however truncation methods need to be better understood and optimized. Besides the Ising model, it can be used for $O(2), O(3)$ spin models, principal chiral models, gauge models (Ising, $U(1)$ and $S U(2))$ [15], $\mathrm{CP}(\mathrm{N})$ models [16], fermions [17, 18]. It would be interesting to make detailed comparisons between this approach and the Tensor Network Matrix Product States approach nicely reviewed during this conference [19]. The TRG method was also used for finitetemperature studies of the gauge Ising model [20]. It has also been used to design quantum simulators, [21, 22, 2, 23] or measure the entanglement entropy [24, 25, 26].

The TRG blocking is simple and exact. We briefly remind the procedure for the 2D Ising model. For each link, we use the character expansion

$$
\exp \left(\beta \sigma_{1} \sigma_{2}\right)=\cosh (\beta) \sum_{n_{12}=0,1}\left(\sqrt{\tanh (\beta)} \sigma_{1} \sqrt{\tanh (\beta)} \sigma_{2}\right)^{n_{12}}
$$


We then regroup the four terms involving a given spin $\sigma_{i}$ and sum over its two values \pm 1 . The results can be expressed in terms of a tensor:

$$
T_{x x^{\prime} y y^{\prime}}^{(i)}=f_{x} f_{x^{\prime}} f_{y} f_{y^{\prime}} \delta\left(\bmod \left[\mathrm{x}+\mathrm{x}^{\prime}+\mathrm{y}+\mathrm{y}^{\prime}, 2\right]\right),
$$

where $f_{0}=1$ and $f_{1}=\sqrt{\tanh (\beta)}$ and $x, x^{\prime}, y, y^{\prime}$ represent the four indices attached to the site. The delta symbol is 1 if $x+x^{\prime}+y+y^{\prime}$ is even and zero otherwise. The partition function can then be rewritten exaclty as:

$$
Z=2^{V}(\cosh (\beta))^{2 V} \operatorname{Tr} \prod_{i} T_{x x^{\prime} y y^{\prime}}^{(i)}
$$

where $\operatorname{Tr}$ mean contractions (sums over 0 and 1) over the links. An important aspect [14] is that the TRG blocking neatly separates the degrees of freedom inside the block (which are integrated over), from those kept to communicate with the neighboring blocks. The blocking procedure defines a new rank-4 tensor $T_{X X^{\prime} Y Y^{\prime}}^{\prime}$ where each index now takes four values:

$$
\begin{aligned}
& T_{X\left(x_{1}, x_{2}\right) X^{\prime}\left(x_{1}^{\prime}, x_{2}^{\prime}\right) Y\left(y_{1}, y_{2}\right) Y^{\prime}\left(y_{1}^{\prime}, y_{2}^{\prime}\right)}^{\prime}= \\
& \sum_{x_{U}, x_{D}, x_{R}, x_{L}} T_{x_{1} x_{U} y_{1} y_{L}} T_{x_{U} x_{1}^{\prime} y_{2} y_{R}} T_{x_{D} x_{2}^{\prime} y_{R} y_{2}^{\prime}} T_{x_{2} x_{D} y_{L} y_{1}^{\prime}},
\end{aligned}
$$

where $X\left(x_{2}, x_{2}\right)$ is a notation for the product states e. g. , $X(0,0)=1, X(1,1)=2, X(1,0)=$ $3, X(0,1)=4$. The partition function can then be rewritten exactly as

$$
Z=2^{V}(\cosh (\beta))^{2 V} \operatorname{Tr} \prod_{2 i} T_{X X^{\prime} Y Y^{\prime}}^{\prime(2 i)}
$$

where $2 i$ denotes the sites of the coarser lattice with twice the lattice spacing of the original lattice. Using a truncation in the number of "states" carried by the indices and rescaling all the tensors in such a way that one of them stays equal to 1, we obtain fixed point equations [14]. The procedure can be extended easily for models with Abelian symmetries, while non-Abelian symmetries seem to require Clesch-Gordan coefficients [15].

The idea of "states" can be made more precise by noticing that the partition function can be expressed in terms of a transfer matrix:

$$
Z=\operatorname{Tr} \mathbb{T}^{L_{t}}
$$

The matrix elements of $\mathbb{T}$ can be written as a product of tensors associated with the sites of a Euclidean time slice (fixed $t$ ) and traced over the space indices. In $1+1$ dimensions [21]:

$$
\mathbb{T}_{\left(n_{1}, n_{2}, \ldots n_{L_{x}}\right)\left(n_{1}^{\prime}, n_{2}^{\prime} \ldots n_{L_{x}}^{\prime}\right)}=\sum_{\tilde{n}_{1} \tilde{n}_{2} \ldots \tilde{n}_{L_{x}}} T_{\tilde{n}_{L_{x}} \tilde{n}_{1} n_{1} n_{1}^{\prime}}^{(1, t)} T_{\tilde{n}_{1} \tilde{n}_{2} n_{2} n_{2}^{\prime} \ldots}^{(2, \ldots)} \ldots T_{\tilde{n}_{L_{x-1}}}^{\left(L_{x}, t\right)} \tilde{n}_{L_{x}} n_{L_{x}} n_{L_{x}}^{\prime} .
$$

For the $O(2)$ model with a chemical potential:

$$
T_{\tilde{n}_{x-1} \tilde{n}_{x} n_{x} n_{x}^{\prime}}^{(x, t)}=\sqrt{I_{n_{x}}\left(\beta_{\tau}\right) I_{n_{x}^{\prime}}\left(\beta_{\tau}\right) I_{\tilde{n}_{x-1}}\left(\beta_{s}\right) I_{\tilde{n}_{x}}\left(\beta_{s}\right) \mathrm{e}^{\left(\mu\left(n_{x}+n_{x}^{\prime}\right)\right)}} \delta_{\tilde{n}_{x-1}+n_{x}, \tilde{n}_{x}+n_{x}^{\prime}} .
$$

The Kronecker delta function reflects the existence of a conserved current which can be associated with the particle number. By taking the time continuum limit, we can use $\mathbb{T}$ to define an Hamiltonian and the eigenstates of $\mathbb{T}$ are then in good approximation Hamiltonian eigenstates. 
The blocking procedure can be used for a single time slice [21]. In the $O(2)$ example, the indices run over the entire positive and negative integers. A good approximation for the microscopic theory can be obtained by just keeping $D_{b}=2 n_{\max }+1$ values ("states") centered at the origin. Starting from this initial approximation and performing blocking without truncation would lead to a complexity growing exponentially like $D_{b}^{N_{s}}$ where $N_{s}$ is the number of spatial sites on a time slice. However if at each step, we keep projecting to a subspace of dimensions $D_{b}$, then the complexity is reduced to a logarithmic growth! However, the errors do not seem to decrease in a uniform way when $D_{b}$ increases and much remains to be understood about this question.

Note also that partially traced density matrices can be used to calculate the entanglement entropy. We consider the subdivision of the system $A B$ into $A$ and $B$ as a subdivision of the spatial indices and define $\hat{\rho}_{A} \equiv \operatorname{Tr}_{B} \hat{\rho}_{A B}$. We can use blocking methods until $A$ and $B$ are each reduced to a single site. The $n$-th order Rényi entanglement entropy is defined as:

$$
S_{n}(A) \equiv \frac{1}{1-n} \ln \left(\operatorname{Tr}\left(\left(\hat{\rho}_{A}\right)^{n}\right)\right) .
$$

This method can be used (for not too large volumes) to calculate the entanglement entropy [24] and verify the predictions of CFT $[25,26]$.

\section{FAQs}

In this section we answer some FAQs related to the tensor formulation. We focus on the case of the $O(2)$ and Ising models. Generalizations and details will be provided in a forthcoming preprint.

\subsection{FAQ1: Do truncation break global symmetries? Answer: No}

We consider the example of the $O(2)$ model. The action and the measure of integration are invariant under the global symmetry $\theta_{\mathbf{x}} \rightarrow \theta_{\mathbf{x}}+\Delta$, this implies

$$
\left\langle f\left(\theta_{\mathbf{x}_{1}}, \ldots, \theta_{\mathbf{x}_{N}}\right)\right\rangle=\left\langle f\left(\theta_{\mathbf{x}_{1}}+\Delta, \ldots, \theta_{\mathbf{x}_{N}}+\Delta\right)\right\rangle .
$$

Assuming that $f$ is $2 \pi$-periodic in all its aguments, it can be expressed in terms of Fourier modes and

$$
\left\langle\exp \left(i\left(n_{1} \theta_{\mathbf{x}_{1}}+\ldots n_{N} \theta_{\mathbf{x}_{N}}\right)\right)\right\rangle=\exp \left(\left(n_{1}+\ldots n_{N}\right) \Delta\right)\left\langle\exp \left(i\left(n_{1} \theta_{\mathbf{x}_{1}}+\ldots n_{N} \theta_{\mathbf{x}_{N}}\right)\right)\right\rangle .
$$

From this we conclude that $\sum_{n=1}^{N} n_{i} \neq 0$ implies $\left\langle\exp \left(i\left(n_{1} \theta_{\mathbf{x}_{1}}+\ldots+n_{N} \theta_{\mathbf{x}_{N}}\right)\right)\right\rangle=0$. A detailed analysis shows that this selection rule is due to the current conservation imposed at each site and is independent of the particular values taken by the tensors. So if we set some of the tensor elements to zero as we do in a truncation, this does not affect the selection rule.

\subsection{FAQ2: Can usual boundary conditions be enforced with tensors? Answer: yes}

The most common boundary conditions for spin models are the periodic boundary conditions (PBC) and open boundary conditions (OBC). These conditions can be encoded in the tensors by identifying possible modifications when we perform the spin/field integration

PBC allow us to keep a discrete translational invariance. This can be enforced by taking the tensors translation invariant and assembled in the same way at every site, link etc. This is the simplest option. 
OBC can be implemented by introducing new tensors that can be placed at the boundary. The only difference for these new tensors is that there are missing links at boundary sites. In all the examples we know, it is possible normalize the tensors in such a way that the missing elements can be taken into account by setting the corresponding missing indices to zero.

It is also possible to define new boundary conditions that only make sense in the reformulation, for instance fixing some of the indices corresponding to missing elements to values different to zero, or summing over these. When dual variables can be introduced to make the current conservation automatic, it is sometimes possible to translate boundary conditions on the dual variables into conditions for the boundary links mentioned above. Similar considerations can also apply for gauge models where boundary links have missing plaquettes.

\subsection{FAQ3: Can the Ising traces be performed with Grassmann variables? Answer: yes}

The local tensor can be incorporated into a "supertensor" which is a function of four Grassmann variables attached to each site. We label these four variables with the indices $\eta_{L}, \eta_{A}, \eta_{B} \eta_{R}$, where $L$ means Left and $R$ means Right. We use a LABR ordering to define

$$
T(\eta) \equiv \sum_{n_{L} n_{R} n_{A} n_{B}=0,1} T_{n_{L} n_{R} n_{A} n_{B}} \eta_{L}^{n_{L}} \eta_{A}^{n_{A}} \eta_{B}^{n_{B}} \eta_{R}^{n_{R}}
$$

where $(\eta)$ is a short notation for $\left(\eta_{L}, \eta_{A}, \eta_{B}, \eta_{R}\right)$. Because of the factorization properties $\left(\mathbb{Z}_{2}\right.$ is abelian):

$$
T_{1111}=T_{1100} \times T_{0011}=T_{0101} \times T_{1010}=T_{1001} \times T_{0110},
$$

the tensor function exponentiates

$$
T(\eta)=\mathrm{e}^{T_{2}(\eta)},
$$

with

$$
T_{2}(\eta)=t_{s} \eta_{L} \eta_{R}+t_{t} \eta_{A} \eta_{B}+\sqrt{t_{s} t_{t}}\left(\eta_{L} \eta_{A}+\eta_{L} \eta_{B}+\eta_{A} \eta_{R}+\eta_{B} \eta_{R}\right)
$$

An simple exponential form of the Grassmannian transfer matrix

$$
\mathbb{T}(\{\eta\}) \equiv \sum_{\{n\}} \mathbb{T}_{\left(n_{A 1}, \ldots, n_{A N_{s}}, n_{B 1}, \ldots, n_{B N_{s}}\right)} \eta_{A 1}^{n_{A 1}} \eta_{B 1}^{n_{B 1}} \ldots \eta_{A N_{s}}^{n_{A N_{s}}} \eta_{B N_{s}}^{n_{B N_{s}}}
$$

can be obtained by inserting $\mathrm{e}^{+\eta_{R 1} \eta_{L 2}}$ between $T\left(\eta_{1}\right)$ and $T\left(\eta_{2}\right)$ and integrating with $\int d \eta_{L 2} d \eta_{R 1}$ and repeating to the next neighbor. If we use periodic boundary conditions in the $\sigma$ formulation, the summation between the last site and the first site requires the insertion of $\mathrm{e}^{-\eta_{R N_{S}} \eta_{L 1}}$ (antiperiodic condition in space). This Gaussian integration can be performed exactly, however the ordering is not the "standard Berezin order". The connection with exact results will be discussed in a forthcoming preprint.

\section{Concluding remarks}

The tensor reformulation of lattice models with compact fields provides an exact discretization of the partition function. We expect that if the truncation is not too drastic, the model should keep its universal properties. This leaves us with a finite system that can be implemented either with a physical device (analog computing) or a commercial quantum computer (digital computing). 
Following the the analog option, we have used the tensor reformulation to propose QC/S of the Abelian Higgs model [2, 23]. This is nicely discussed in J. Unmuth-Yockey's talk which should appear in these Proceedings. We have reformulated the lattice Abelian Higgs model (scalar QED) in $1+1$ dimension in a manifestly gauge invariant way that connects smoothly the classical Lagrangian formulation used by lattice gauge theorists and the quantum Hamiltonian method used in condensed matter. Calculations of the Polyakov loop show a remarkable data collapse that survives the time continuum limit. This collapse could be tested with small volumes. We proposed to use a BoseHubbard (BH) Hamiltonian on a ladder as a quantum simulator for this model. Johannes Zeiher has designed methods to possibly implement such a ladder structure with equipment available in Immanuel Bloch's lab [27]. These two points are illustrated in Fig. 1.

5 states ladder with 9 rungs

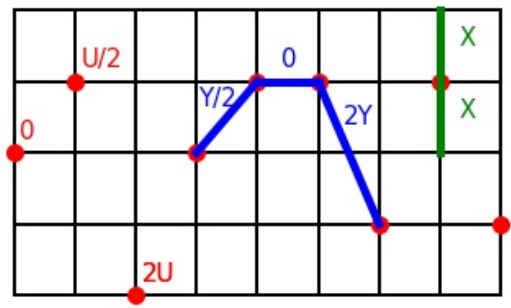

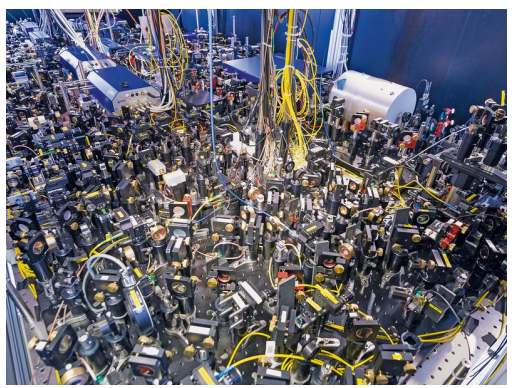

Figure 1: Left: a ladder with one atom per rung: there is tunneling along the vertical direction but not in the horizontal direction, however there are short range attractive interactions. A parabolic potential is applied in the spin (vertical) direction. With a color code corresponding to the picture: $\bar{H}=\frac{\tilde{U}_{g}}{2} \sum_{i}\left(\bar{L}_{(i)}^{z}\right)^{2}+$ $\frac{\tilde{Y}}{2} \sum_{i}\left(\bar{L}_{(i)}^{z}-\bar{L}_{(i+1)}^{z}\right)^{2}-\tilde{X} \sum_{i} \bar{L}_{(i)}^{x}$. Right: an optical lattice experiment of I. Bloch's group [27].

Analog simulations are good to provide a large number of identical degrees of freedom and are typically good to probe universal properties. Better local control could be achieved with quantum computers. The space-time formulation of the TRG provide inspirational ways to "Trotterize" the time evolution at real time. It is tempting to force analog devices to evolve sequentially, especially if the interactions can only be applied for a short time. It would also be very interesting to compare compact and non-compact models. In the non-compact case, a field cut needs to be introduced $[7,10]$. Note that this makes the perturbative series converge [28].

Acknowledgements: This research was supported in part by the Dept. of Energy under Award Numbers DE-SC0010113 and DE-SC0019139. We thank Alexei Bazavov, Philipp Preiss, Shan-Wen Tsai, Judah Unmuth-Yockey, Li-Ping Yang, Johannes Zeiher and Jin Zhang who have contributed to part of the reviewed material in sections 2 and 4 .

\section{References}

[1] V. Kasper, F. Hebenstreit, F. Jendrzejewski, M. K. Oberthaler, and J. Berges, New J. Phys., vol. 19, no. 2, p. 023030, 2017. 
[2] J. Zhang, J. Unmuth-Yockey, A. Bazavov, S. W. Tsai, and Y. Meurice, "Quantum simulation of the universal features of the Polyakov loop," arXiv:1803.11166, 2018.

[3] E. A. Martinez et al., vol. 534, pp. 516-519, 2016.

[4] U.-J. Wiese, Ann. Phys. (Berlin), vol. 525, pp. 777-796, 2013.

[5] E. Zohar, J. I. Cirac, and B. Reznik, Rep. Prog. Phys., vol. 79, no. 1, p. 014401, 2016.

[6] M. Dalmonte and S. Montangero, Contemp. Phys., vol. 57, no. 3, pp. 388-412, 2016.

[7] S. P. Jordan, K. S. M. Lee, and J. Preskill, Science, vol. 336, pp. 1130-1133, 2012.

[8] N. Klco, E. F. Dumitrescu, A. J. McCaskey, T. D. Morris, R. C. Pooser, M. Sanz, E. Solano, P. Lougovski, and M. J. Savage, Phys. Rev. A, vol. 98, no. 3, p. 032331, 2018.

[9] H. Lamm and S. Lawrence, Phys. Rev. Lett., vol. 121, no. 17, p. 170501, 2018.

[10] N. Klco and M. J. Savage, “Digitization of Scalar Fields for NISQ-Era Quantum Computing,” arXiv:1808.10378, 2018.

[11] M. Levin and C. P. Nave, Phys. Rev. Lett., vol. 99, p. 120601, Sep 2007.

[12] Z.-C. Gu, M. Levin, B. Swingle, and X.-G. Wen, Phys. Rev. B, vol. 79, p. 085118, Feb 2009.

[13] Z. Y. Xie, J. Chen, M. P. Qin, J. W. Zhu, L. P. Yang, and T. Xiang, Phys. Rev. B, vol. 86, p. 045139, Jul 2012.

[14] Y. Meurice, Phys. Rev., vol. B87, no. 6, p. 064422, 2013.

[15] Y. Liu, Y. Meurice, M. P. Qin, J. Unmuth-Yockey, T. Xiang, Z. Y. Xie, J. F. Yu, and H. Zou, Phys. Rev., vol. D88, p. 056005, 2013.

[16] H. Kawauchi and S. Takeda, Phys. Rev., vol. D93, no. 11, p. 114503, 2016.

[17] Y. Shimizu and Y. Kuramashi, Phys. Rev., vol. D97, no. 3, p. 034502, 2018; and refs. therein.

[18] R. Sakai, S. Takeda, and Y. Yoshimura, PTEP, vol. 2017, no. 6, p. 063B07, 2017.

[19] M. C. Bañuls, K. Cichy, J. I. Cirac, K. Jansen, and S. Kühn, "Tensor Networks and their use for Lattice Gauge Theories,” arXiv:1810.12838, 2018 and refs. therein, these Proceedings.

[20] Y. Kuramashi and Y. Yoshimura, "Three-dimensional finite temperature $\mathrm{Z}_{2}$ gauge theory with tensor network scheme," arXiv:1808:08025, 2018; and these Proceedings.

[21] H. Zou, Y. Liu, C.-Y. Lai, J. Unmuth-Yockey, A. Bazavov, Z. Y. Xie, T. Xiang, S. Chandrasekharan, S. W. Tsai, and Y. Meurice, Phys. Rev., vol. A90, no. 6, p. 063603, 2014.

[22] A. Bazavov, Y. Meurice, S.-W. Tsai, J. Unmuth-Yockey, and J. Zhang, Phys. Rev. D, vol. 92, p. 076003, Oct 2015.

[23] J. Unmuth-Yockey, J. Zhang, A. Bazavov, Y. Meurice, and S.-W. Tsai, "Universal features of the Abelian Polyakov loop in 1+1 dimension,” arXiv:1807.09186, 2018; and these Proceedings.

[24] L.-P. Yang, Y. Liu, H. Zou, Z. Y. Xie, and Y. Meurice, Phys. Rev., vol. E93, no. 1, p. 012138, 2016.

[25] J. Unmuth-Yockey, J. Zhang, P. M. Preiss, L.-P. Yang, S. W. Tsai, and Y. Meurice, Phys. Rev., vol. A96, no. 2, p. 023603, 2017.

[26] A. Bazavov, Y. Meurice, S. W. Tsai, J. Unmuth-Yockey, L.-P. Yang, and J. Zhang, Phys. Rev., vol. D96, no. 3, p. 034514, 2017.

[27] https://www.quantum-munich.de/research/single-site-detection-and-manipulation-in-optical-lattices/

[28] Y. Meurice, Phys. Rev. Lett., vol. 88, p. 141601, Mar 2002. 